\begin{tabular}{|c|c|c|c|}
\hline Isan Vehar, MD & Marina Boushra, MD & Prince Ntiamoah, MD & hl, MD, MS \\
\hline $\begin{array}{l}\text { epartment of Critical Care Medicine; } \\
\text { epartment of Pulmonary Medicine; } \\
\text { eveland Clinic. Cleveland } \mathrm{OH}\end{array}$ & $\begin{array}{l}\text { Department of Critical Care Medicine, } \\
\text { Cleveland Clinic, Cleveland, OH }\end{array}$ & $\begin{array}{l}\text { Department of Critical Care Medicine; } \\
\text { Department of Pulmonary Medicine; } \\
\text { Cleveland Clinic Cleveland } \mathrm{OH}\end{array}$ & $\begin{array}{l}\text { Department of Critical Care Medicine; } \\
\text { Department of Pulmonary Medicine; } \\
\text { Cleveland Clinic Cleveland, } \mathrm{OH}\end{array}$ \\
\hline
\end{tabular}

\title{
Post-acute sequelae of SARS-CoV-2 infection: Caring for the 'long-haulers'
}

\section{ABSTRACT}

An estimated 10\% of COVID-19 survivors continue to experience symptoms several weeks to months after the appearance of initial symptoms, a condition termed postacute sequelae of SARS-CoV-2 infection (PASC). These patients, also called "long-haulers," most commonly report protracted symptoms of fatigue, cough, dyspnea, chest tightness, difficulty concentrating, arthralgia, olfactory dysfunction, and headache. While age, comorbid medical conditions, and COVID-19 severity are risk factors, young and previously healthy individuals with mild COVID-19 are also at risk. Recognition of symptoms, evaluation, supportive treatment, and attention to medical comorbidities are the cornerstones of medical management.

\section{KEY POINTS}

If a patient has COVID-19 symptoms at 4 weeks, assess for pulmonary, cardiac, neurocognitive, and psychiatric complications.

In patients with PASC, symptoms may persist for more than 60 days and as long as 6 months.

Focus treatment on managing comorbidities, pulmonary rehabilitation, and continued follow-up.

\begin{abstract}
s the COVID-19 pandemic continues A globally, there is increasing need to understand the entire disease spectrum and anticipate the long-term management of survivors. Early in the pandemic, adults with mild to moderate COVID-19 were believed to have a short-term course of acute illness lasting approximately 2 weeks, after which symptoms completely resolved. However, emerging data have described a subgroup of patients with a prolonged course of symptoms lasting several weeks to months. ${ }^{1}$
\end{abstract}

See related article, page 273

This protracted form of COVID-19 has been given several descriptive names, including the post-acute COVID-19 syndrome, long COVID, and long-haul COVID-19, with affected patients termed "long-haulers." But most recently, the National Institutes of Health $(\mathrm{NIH})$ called for a consensus terminology, ie, the post-acute sequelae of SARSCoV-2 infection (PASC). ${ }^{2}$ Although the definition seems to be evolving and is not yet formalized, we currently recognize PASC if symptoms persist for at least 28 days after the onset of COVID-19 symptoms. By building a consensus definition and terminology for PASC, the NIH aims to unify an initiative including $\$ 1.15$ billion in grant funding over the course of 4 years to investigate PASC and, hopefully, determine how to treat it. ${ }^{2}$

PASC should be recognized among other sequelae affecting COVID-19 survivors. For example, the postintensive care syndrome (PICS) describes a distinct group of patients who develop new or worsening cognitive, physical, or psychiatric health impairments after suffering critical COVID-19 symptoms 
TABLE 1

\section{Prevalence of COVID-19 symptoms in a series of 143 cases}

\begin{tabular}{lcc} 
& \multicolumn{2}{c}{$\%$ of patients } \\
\cline { 2 - 3 } Symptom & At onset & At 60 days \\
\hline Fatigue & 79 & 56 \\
Dyspnea & 67 & 42 \\
Joint pain & 56 & 26 \\
Chest pain & 40 & 22 \\
Cough & 70 & 18 \\
Anosmia & 43 & 17 \\
Sicca symptoms & 22 & 16 \\
Rhinitis & 32 & 12 \\
Red eyes & 27 & 10 \\
Dysgeusia & 50 & 10 \\
Headache & 50 & 8 \\
Sputum production & 18 & 7 \\
Lack of appetite & 56 & 7 \\
Sore throat & 30 & 6 \\
Vertigo & 18 & 5 \\
Myalgia & 56 & 5 \\
Diarrhea & 30 & 2 \\
& Data from Carfi et al, reference 6.
\end{tabular}

requiring admission to the intensive care unit (ICU). ${ }^{3}$ In contrast, PASC applies to the broad range of COVID-19 survivors, from those with mild acute illness who may have never required hospitalization to ICU survivors. Survivors of critical illness associated with COVID-19 can be viewed as likely having an overlap of PICS and PASC as part of the spectrum of postCOVID-19 complications.

\section{TRUE NUMBERS ARE HARD TO ASSESS}

A marked variability in reported symptoms, duration, and definitions of COVID-19 sequelae in studies makes it difficult to estimate the true incidence of PASC. However, by some estimates, at least $10 \%$ of patients who test positive for COVID-19 experience symptoms for longer than 3 weeks. ${ }^{4}$

Although advanced age, obesity, comorbid psychiatric conditions, and other chronic medical conditions are risk factors for PASC, nearly $20 \%$ of suspected cases are in adults ages 18 to 34 with no chronic medical conditions. ${ }^{5}$ Studies that included hospitalized patients report an even higher prevalence of protracted symptoms, with data suggesting more than two-thirds of these patients have continued symptoms 6 months after recovery from acute COVID-19. ${ }^{6,7}$ We have insufficient data to directly attribute the prolonged symptoms in this population to PASC rather than to other causes of protracted symptoms in hospitalized patients, such as PICS. The prolonged symptoms are likely multifactorial and may be difficult to attribute to a single cause.

\section{MECHANISMS PROPOSED, BUT PATHOPHYSIOLOGY UNKNOWN}

The pathophysiology of the PASC is not known. Clinicians and researchers are exploring the possibilities of a persistent hyperinflammatory state, inadequate antibody response, ongoing viral activity, and organ damage as a reflection of acute insult from the infectious phase. ${ }^{8}$ Furthermore, it is likely that this syndrome represents a multifactorial presentation attributable to symptoms of underlying medical conditions, features of the acute disease state, and symptoms associated with physical deconditioning from precautionary isolation measures and acute illness. ${ }^{8}$

Despite the chronicity of symptoms, the US Centers for Disease Control and Prevention reports that most immunocompetent patients with mild to moderate COVID-19 are unlikely to be contagious at 10 days after symptom onset, or at 20 days for most immunocompromised patients and patients with severe illness, which allows for discontinuation of isolation precautions at those time points. ${ }^{9,10}$

\section{A RANGE OF SYMPTOMS}

Data on symptoms of PASC commonly include outpatients with mild to moderate disease severity, but many studies have included patients with severe COVID-19 who required hospitalization with or without ICU admission. ${ }^{1,8}$ Most studies to date have recognized symptoms consistent with PASC as persisting after at least 14 to 21 days. Emerging reports are recognizing PASC as persisting symptoms at 28 days in COVID-19 survivors, although an official defi- 
nition has not been clearly delineated.

The most commonly reported symptoms are fatigue, cough, shortness of breath, chest pain, difficulty concentrating, arthralgia, lowgrade fever, and headache (Table 1). ${ }^{6-8,11}$ Other reported symptoms include cognitive impairment ("brain fog"), olfactory and gustatory dysfunction, sleep difficulty, depression, anxiety, gastrointestinal upset, rashes, alopecia, and palpitations.,6 Persistent dermatologic manifestations have been described: in a multinational study of patients with dermatologic manifestations, pernio ("COVID toes") was observed in 103 patients, and persisted at 60 days after diagnosis in $7 \%$ of them. ${ }^{12}$

Two studies noted symptoms at the 60-day follow-up in more than two-thirds of patients recovering from COVID-19., ${ }^{6,7}$ In a study from Italy, Carfi et $\mathrm{al}^{6}$ found that at 60 days after onset of COVID-19 symptoms, only 18 (12.6\%) of 143 patients were completely free of any COVID-19-related symptom, $32 \%$ had 1 or 2 symptoms, and $55 \%$ had 3 or more symptoms. Other studies have shown at least 1 persistent symptom, most commonly fatigue or dyspnea, in more than half of patients at 110 days or 180 days, suggesting the longest duration has yet to be determined. ${ }^{13,14}$

Huang et $\mathrm{al}^{14}$ reporting on patients 6 months after their acute COVID-19 diagnosis, noted continued fatigue in 63\%, sleep difficulties in $24 \%$, and anxiety or depression in $23 \%$. The level of persistent lung diffusion impairment and exercise intolerance at 6 months correlated with the severity of COVID-19 illness.

Of note, PASC affects patients across the spectrum of disease severity. Garrigues et $\mathrm{al}^{13}$ found no statistically significant difference in the symptoms reported in patients with COVID-19 requiring hospitalization compared with those requiring admission to an ICU. On the other hand, a study from the United Kingdom found nearly twice the rate of psychological distress in patients who required ICU admission compared with those admitted to a general ward. ${ }^{11}$

In a study of outpatients with COVID-19, $35 \%$ had not returned to their baseline health 2 to 3 weeks following positive testing for SARS-CoV-2. ${ }^{5}$ This trend of protracted symptoms was consistent even in previously healthy patients: approximately $20 \%$ of those ages 18 to 34 without chronic medical conditions who were diagnosed with mild COVID-19 not requiring hospitalization reported that they had not returned to their baseline state of health 2 to 3 weeks after testing. ${ }^{5}$ Even patients with dermatologic-dominant and otherwise mild COVID-19 have been reported to manifest dermatologic signs of pernio or livedo reticularis for as long as 150 days from initial diagnosis, again demonstrating the range of initial disease severity that variably becomes PASC. 12

PASC has also had significant global economic impact. A single-center study in France reported that more than $25 \%$ of previously active workers $(n=41)$ discharged from the hospital ward (without ICU stay) had not returned to work after 110 days (mean 110.9 days). ${ }^{13}$ An observational cohort study of patients discharged after recovery from COVID-19 in Michigan showed that $40 \%$ of previously employed individuals had not returned to work, and that another $15 \%$ returned with reduced hours or responsibilities at 60-day follow-up. ${ }^{15}$

\section{EVALUATION OF LONG-HAULERS}

Guidelines for evaluation of PASC are being developed. Several large centers have created post-COVID-19 clinics that offer a multidisciplinary approach to evaluation and management, including follow-up for noncritically ill patients and post-ICU care. Most clinics accept COVID-19 patients referred for persistent symptoms 1 month after symptom onset. Most use multiple screening measures, including the Montreal Cognitive Assessment, Hospital Anxiety and Depression Scale, and Impact of Event Scale-6, in addition to medication reconciliation, screening for rehabilitation needs, and pulmonary function testing. ${ }^{16}$ A Post-COVID-19 Functional Status Scale has been created to grade the severity of symptoms, but it has not been validated or widely implemented. ${ }^{17}$

Multidisciplinary care teams typically include primary care, pulmonology, cardiology, infectious disease, neuropsychiatry, behavioral health, social work, physical and occupational therapy, pharmacy, and case management, but their involvement will vary depending on the particular needs of the patient. 


\section{COVID-19 diagnosis}

Arrange for multidisciplinary follow-up

Inpatients: ambulatory oximetry, physical therapy,

occupational therapy, care coordination, home healthcare

Outpatients: primary care follow-up, referral to local COVID-19

hotline

4 weeks after diagnosis or hospital discharge

No persistent symptoms

Resume routine outpatient care

\section{Persistent symptoms}

In-person visit with primary care physician or post-COVID-19 clinic: chest $x$-ray, spirometry, diffusing capacity for carbon monoxide, psychiatric screening, ${ }^{b}$ neurocognitive screening ${ }^{c}$

If pulmonary embolism diagnosed with COVID-19: echocardiogram, electrocardiogram, ventilation-perfusion scan

For all patients, also consider echocardiogram, electrocardiogram

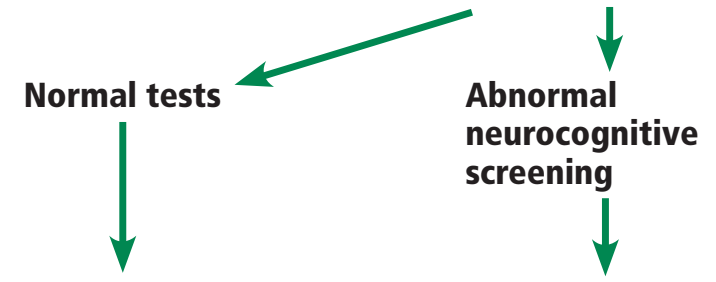

Consider alternative diagnoses for symptoms Refer to post-COVID-19 clinic, if available Optimize comorbid conditions Supportive symptomatic care

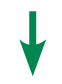

Abnormal chest x-ray spirometry, diffusion capacity, ventilationperfusion scan $\checkmark$

Computed tomography/ computed tomographic pulmonary arteriography

Pulmonary referral

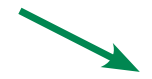

Abnormal echocardiogram and electrocardiogram

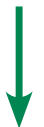

Cardiology referral

aScreening tools to consider: Post-COVID-19 Functional Status Scale, COVID-19 Yorkshire Rehabilitation Screen, University of Pennsylvania Post-COVID Screening Measures.

${ }^{\mathrm{b}}$ Available psychiatric screening tools: General Anxiety Disorder-7 (GAD-7), Patient Health Questionnaire-9 (PHQ-9; for depression screening), PTSD Checklist for DSM-5 (PCL-5), Impact of Event Scale-6 (IES-R; for PTSD screening), Hospital Anxiety and Depression Score (HADS).

'Available neurocognitive screening tools: Montreal Cognitive Assessment (MoCA), Mini-Mental State Examination (MMSE), Cognitive Assessment Tool Rapid Version (CAT-rapid).

Figure 1. Care pathway for patients with the post-acute sequelae of SARS-CoV-2 infection.

The British Thoracic Society ${ }^{18}$ published a guidance algorithm suggesting a follow-up chest radiograph at the 12-week follow-up. However, the timing of imaging tests must be tailored to the individual patient, with some algorithms suggesting chest radiography or computed tomography at 1 month. Our approach to follow-up evaluation and care is shown in Figure 1.

Evaluation of persistent or changing symp- toms in patients recovering from COVID-19 should be done comprehensively but also judiciously. COVID-19 may unmask or exacerbate underlying disease processes such as chronic lung disease or cardiovascular disease, and may serve as motivation to seek medical care for patients who may not typically schedule routine visits. Basic management of chronic medical conditions is important. 


\section{SPECIFIC TREATMENT FOR SYMPTOMS AND COMORBID CONDITIONS}

At present, there are no specific treatments for PASC. But recognizing this syndrome is a key step toward seeking targeted treatment.

Management focuses on specific treatment of the most bothersome symptoms, such as fatigue or cognitive impairment, although efficacy data are lacking. Given the complexity and chronicity of the associated symptoms and their impact on several major organ systems, frequent routine follow-up, establishing rapport, and involving a patient's support system serve as the foundation of management in these patients. A comprehensive, multidisciplinary approach that incorporates detailed management of comorbid medical conditions is the cornerstone of care. Referral to a dedicated post-COVID-19 clinic is recommended, if available.

\section{LIMITATIONS OF CURRENT DATA, AND LESSONS LEARNED}

There are significant limitations in the research efforts to understand the natural history of a pandemic that is merely 1 year old. Limitations include the relatively short time frame of follow-up and a heavy reliance on survey-reporting due to infection-prevention measures that limit in-person follow-up visits.

Reflecting on lessons learned from the out- breaks of severe acute respiratory distress syndrome (SARS) and Middle East respiratory syndrome (MERS), both caused by related coronaviruses, clinicians should anticipate at least a similar range of long-term effects. Survivors of SARS and MERS have been found to have persistent respiratory compromise with abnormal pulmonary function test results, abnormal chest imaging, increased prevalence of psychological conditions, and fatigue that lasted several months. ${ }^{19}$ Mainstays of their treatment included management of comorbid conditions, pulmonary rehabilitation, and ongoing multidisciplinary follow-up aimed at recognizing impairments and improving outcomes.

\section{FUTURE DIRECTIONS}

As part of the PASC initiative, the NIH committed more than $\$ 1$ billion in grant funding toward several research initiatives to streamline efforts of the medical and scientific communities to improve our understanding and treatment of PASC. The SARS-CoV-2 Recovery Cohort will be established as a core resource to help investigate PASC as an important public health concern.

\section{DISCLOSURES}

The authors report no relevant financial relationships which, in the context of their contributions, could be perceived as a potential conflict of interest.

\section{REFERENCES}

1. Infectious Diseases Society of America. Post COVID/long COVID. Updated January 20, 2021. Accessed March 30, 2021. https://www.idsociety.org/covid-19-real-time-learning-network/disease-manifestations--complications/post-covid-syndrome/\#: : :text=In summary\%3A Preliminary reports, moderate-to-severe disease

2. Collins FS. NIH launches new initiative to study "Long COVID." National Institutes of Health. February 23, 2021. Accessed March 30, 2021. https://www.nih.gov/about-nih/who-we-are/nih-director/statements/nih-launches-new-initiative-study-long-covid

3. Biehl M, Sese D. Post-intensive care syndrome and COVID-19implications post pandemic. Cleve Clin J Med 2020 Aug 5. doi:10.3949/ccjm.87a.ccc055

4. Greenhalgh T, Knight M, A'Court C, Buxton M, Husain L. Management of post-acute covid-19 in primary care. BMJ 2020; 370:m3026. doi:10.1136/bmj.m3026

5. Tenforde MW, Kim SS, Lindsell CJ, et al. Symptom duration and risk factors for delayed return to usual health among outpatients with COVID-19 in a multistate health care systems network - United States, March-June 2020. MMWR Morb Mortal Wkly Rep 2020; 69(30):993-998. doi:10.15585/mmwr.mm6930e1

6. Carfi A, Bernabei R, Landi F. Persistent symptoms in patients after acute COVID-19 [Letter]. JAMA 2020; 324(6):603-605. doi:10.1001/jama.2020.12603
7. Carvalho-Schneider C, Laurent E, Lemaignen A, et al. Follow-up of adults with noncritical COVID-19 two months after symptom onset. Clin Microbiol Infect 2021; 27(2):258-263. doi:10.1016/j.cmi.2020.09.052

8. Centers for Disease Control and Prevention. Late sequelae of COVID-10. Updated November 13, 2020. Accessed March 30, 2020. https://www.cdc.gov/coronavirus/2019-ncov/hcp/clinical-care/latesequelae.html

9. Centers for Disease Control and Prevention. Duration of isolation \& precautions for adults with COVID-19. Updated February 13, 2021. Accessed March 30, 2021. https://www.cdc.gov/coronavirus/2019ncov/hcp/duration-isolation.html

10. Centers for Disease Control and Prevention. Discharging COVID-19 patients. Updated February 16, 2021. Accessed March 30, 2021. https://www.cdc.gov/coronavirus/2019-ncov/hcp/disposition-hospitalized-patients.html

11. Halpin SJ, Mclvor C, Whyatt G, et al. Postdischarge symptoms and rehabilitation needs in survivors of COVID-19 infection: a crosssectional evaluation. J Med Virol 2021; 93(2):1013-1022. doi:10.1002/jmv.26368

12. McMahon DE, Gallman AE, Hruza GJ, et al. Long COVID in the skin: a registry analysis of COVID-19 dermatological duration. Lancet 2021; 21(3):313-314. doi:10.1016/S1473-3099(20)30986-5

13. Garrigues $E$, Janvier $P$, Kherabi $Y$, et al. Post-discharge persistent symptoms and health-related quality of life after hospitalization for 


\section{COVID SEQUELAE}

COVID-19. J Infect 2020; 81(6):e4-e6. doi:10.1016/j.jinf.2020.08.029

14. Huang $C$, Huang $L$, Wang $Y$, et al. 6-month consequences of $\mathrm{CO}$ VID-19 in patients discharged from hospital: a cohort study. Lancet 2021; 397(10270):220-232. doi:10.1016/S0140-6736(20)32656-8

15. Chopra V, Flanders SA, O'Malley M, Malani AN, Prescott HC. Sixtyday outcomes among patients hospitalized with COVID-19. Ann Intern Med 2020; Nov 11:M20-5661. doi:10.7326/M20-5661

16. Penn Medicine. Post-COVID Assessment and Recovery Clinic. Accessed March 30, 2021. https://www.pennmedicine.org/for-healthcare-professionals/for-physicians/covid-information/post-covid19assessment-and-recovery-clinic-at-penn

17. Klok FA, Boon GJAM, Barco S, et al. The Post-COVID-19

Functional Status scale: a tool to measure functional status over time after COVID-19. Eur Respir J 2020; 56(1):2001494. doi:10.1183/13993003.01494-2020

18. British Thoracic Society. British Thoracic Society guidance on respiratory follow up of patients with a clinico-radiological diagnosis of COVID-19 pneumonia. May 11, 2020. Accessed March 30, 2021. https://www.brit-thoracic.org.uk/document-library/quality-improvement/covid-19/resp-follow-up-guidance-post-covid-pneumonia/

19. Ahmed H, Patel K, Greenwood D, et al. Long-term clinical outcomes in survivors of severe acute respiratory syndrome and Middle East respiratory syndrome coronavirus outbreaks after hospitalisation or ICU admission: a systematic review and meta-analysis. J Rehabil Med 2020; 52(5):jrm00063. doi:10.2340/16501977-2694

Address: Susan Vehar, MD, A90, Cleveland Clinic, 9500 Euclid Avenue, Cleveland, $\mathrm{OH} 44195 ;$ vehars@ccf.org 